\title{
Calculation of Shape Anisotropy for Micropatterned Thin Fe-Ni Films for On-Chip RF Applications
}

\author{
Marina Vroubel, Yan Zhuang, Behzad Rejaei, Joachim N. Burghartz, Ankur M. Crawford, and Shan X. Wang
}

\begin{abstract}
We present a self-consistent calculation of the effective anisotropy of patterned, integrated ferromagnetic films. For comparison, experimental data were obtained from electroplated thin $(0.5 \mu \mathrm{m}) \mathrm{Ni}-\mathrm{Fe}$ films with different patterns. The effective anisotropy of patterned samples was increased by shape demagnetizing effects from just 10 Oe up to 100 Oe. The samples with suitable characteristics were subsequently used as cores for on-chip inductors and microstrips.
\end{abstract}

Index Terms-Demagnetization, magnetic anisotropy, magnetic permeability, magnetic thin films.

\section{INTRODUCTION}

$\mathbf{T}$ HE low ferromagnetic resonance (FMR) frequency of ferromagnetic (FM) films compatible with the current standard integrated circuit technology prohibits their application in integrated RF devices such as inductors and transformers. Film micropatterning has been suggested as the means to raise the FMR frequency by increasing the magnetic shape anisotropy [1], [2]. This, in turn, requires accurate models, which predict the nonuniform distribution of magnetization and demagnetization field inside the film [3]. The well-known perturbation theoretical calculations in the literature [4], however, assume a large, additionally applied DC magnetic field, which cannot be realized in IC applications. The magnetometric approximation [5], on the other hand, does not use a DC field, but assumes a uniform magnetization in the whole sample. This approach looses its validity in on-chip designs were nonellipsoidal (rectangular) samples are used. In this work we present a full, self-consistent calculation of the nonuniform distribution of the magnetization and demagnetization field in patterned ferromagnetic thin films. We will investigate the influence of patterning on the initial (DC) permeability and FMR frequency of the films. The results obtained are shown to be in good agreement with B-H loop measurements on patterned samples.

Manuscript received October 16, 2003. The work of M. Vroubel and Y Zhuang was supported by the Foundation for Fundamental Research on Matter (FOM), the Netherlands.

M. Vroubel, Y. Zhuang, B. Rejaei, and J. N. Burghartz are with Laboratory of Electronic Components, Technology and Materials, Delft Institute of Microelectronics and Submicron Technology (DIMES), Delft University of Technology, 2628 CT Delft, The Netherlands (e-mail: m.vroubel@its.tudelft.nl; Y.Zhuang@dimes.tudelft.nl; b.rejaei@ewi.tudelft.nl; burgh@ dimes.tudelft.nl).

A. M. Crawford and S. X. Wang are with Department of Material Science and Engineering, Stanford University, Stanford, CA 94305 USA (e-mail Ankur.Mohan@Stanford.edu; sxwang@ee.stanford.edu).

Digital Object Identifier 10.1109/TMAG.2004.835096

\section{MODEL}

For a thin FM film with a uniform easy axis lying in the plane of the film (along the $z$ axis) and a uniform demagnetization field, the FMR frequency is given by the Kittel formula

$$
\omega_{\mathrm{FMR}}^{2}=\gamma^{2}\left[H_{k}+\left(N_{x}-N_{z}\right) M_{z}\right]\left[H_{k}+\left(N_{y}-N_{z}\right) M_{z}\right]
$$

where $\gamma$ is the gyromagnetic ratio, $M$ is the magnetization, $H_{k}$ is the intrinsic anisotropy field, and $N_{x}, N_{y}, N_{z}$ are the demagnetization factors in the $x, y$, and $z$ directions, respectively, and the $y$ axis is perpendicular to the plane. For a thin ferromagnetic film magnetized along the in-plain easy axis, the condition $N_{y} \approx 1 \gg N_{z}$ holds. Since for Ni-Fe $H_{k} \ll M_{z}$, (1) can be simplified to

$$
\omega_{\mathrm{FMR}}^{2}=\gamma^{2} M_{z}\left\{H_{k}+\left(N_{x}-N_{z}\right) M_{z}\right\}=\gamma^{2} M_{z} H_{k, \mathrm{eff}}
$$

Typical values of $H_{k}$ for $\mathrm{Ni}_{78} \mathrm{Fe}_{22}$ alloys are in the range 2-10 Oe, which yield a maximum FMR frequency of $0.9 \mathrm{GHz}$ for in-plane infinite thin films.

In traditional, discrete devices the FMR frequency is increased by applying an additional DC field along the easy axis, effectively increasing the anisotropy $H_{k}$. This approach is not feasible for on-chip applications, where permanent magnets or bulky DC coils cannot be used. Instead, one has to exploit the demagnetization field induced by the shape anisotropy of patterned films [1], [2]. One should bear in mind, however, that the concept of demagnetization coefficients was introduced for uniform demagnetizing fields of an ellipsoidal body, and cannot be applied to on-chip designs were rectangular samples are used. In the latter case, the demagnetization field becomes nonuniform, leading to a nonuniform alignment of the magnetization.

In order to obtain the distribution of magnetization inside patterned rectangular samples, we consider the total magnetic energy of the system consisting of the demagnetizing, anisotropy, and external-field energies (the exchange energy is neglected)

$$
E=\int\left[\frac{1}{2} \vec{M}(\vec{r}) \cdot \vec{H}_{d}(\vec{r})-K_{M} M_{z}^{2}(\vec{r})-\vec{H}_{\mathrm{DC}} \cdot \vec{M}(\vec{r})\right] d V
$$

where $K_{M}$ is the anisotropy coefficient, $\vec{H}_{\mathrm{DC}}$ is an external DC field, and $\vec{H}_{\mathrm{dem}}$ is the demagnetization field given by

$$
\vec{H}_{\mathrm{dem}}(\vec{r})=\vec{\nabla} \int_{V^{\prime}} \frac{\left(\vec{r}^{\prime}-\vec{r}\right)}{\left|\vec{r}^{\prime}-\vec{r}\right|^{3}} \cdot \vec{M}\left(\vec{r}^{\prime}\right) d V^{\prime} .
$$


We will assume that the magnetization is uniform along the normal to the thin film (along the $y$ axis) and has only in-plane components. This is a realistic assumption for thin $(<1 \mu \mathrm{m})$ ferromagnetic films with in-plane anisotropy in case of in-plane DC fields. The energy functional (3) can be discretized by dividing the sample into $N_{p}$ rectangular prisms of equal size. Assuming a constant magnetization within each cell and neglecting the nondiagonal components of the demagnetization fields, the energy functional can then be written as

$$
\begin{aligned}
E=\frac{1}{2} \sum_{i, j=1}^{N_{p}}\left(M_{x}^{i} N_{x x}^{i j} M_{x}^{j}+M_{z}^{i} N_{z z}^{i j} M_{z}^{j}\right) & \\
& -\sum_{i=1}^{N_{p}}\left(K_{M}\left(M_{z}^{i}\right)^{2}+\vec{H}_{\mathrm{DC}} \cdot \vec{M}^{i}\right)
\end{aligned}
$$

where $\vec{M}^{i}$ denotes the magnetization in the $i$ th cell, and $N_{x x}^{i j}$ and $N_{z z}^{i j}$ are the magnetometric demagnetization tensors representing the (averaged) demagnetization field in the cell $i$ arising from the magnetization of the cell $j$ [5]. Equation (5) was numerically minimized under the constraint $|\vec{M}|=$ const for various patterned samples.

\section{EXPERIMENTAL RESULTS}

Fig. 1 shows the measured hard-axis B-H loops for three patterned Ni-Fe films (Table I). The effective anisotropy $H_{k \text {,eff }}$ is extracted from the initial slope of the B-H loop

$$
\frac{H_{k, \text { eff }}}{M}=\frac{H_{\mathrm{DC}, x}}{M_{x}} .
$$

Although originally derived for uniformly magnetized samples [6], (6) can be applied to nonuniform samples in a phenomenological way. For samples A, B, and C (Table I), $H_{k \text {,eff was found }}$ to be 45,100 , and $35 \mathrm{Oe}$, respectively, corresponding to a FMR frequency of 1.9, 2.8, and $1.65 \mathrm{GHz}$. The latter was verified by using the samples as cores of on-chip inductors and microstrips [7], [8]. The estimated DC permeability (initial slope of the B-H curve for a small external field) was 210,100 , and 260 for samples A, B, and C, respectively.

The results of our self-consistent calculations are shown as solid black lines in Fig. 1. In addition, we have included the values predicted by the magnetometric approach [5], which assumes a uniform magnetization, but averages the demagnetization field to arrive at overall demagnetization factors. As shown in Fig. 1, the latter method overestimates the effective anisotropy and underestimates the value of the initial permeability in all cases.

The self-consistent model, however, shows a much better agreement with experiments, in particular for samples with a large shape anisotropy (A,B). Furthermore, both experimental curves and the self-consistent calculation show full saturation along the hard axes to take place at much higher fields than those predicted by the magnetometric approach. Fig. 2 shows the calculated distribution of the $x$ component of magnetization in sample $\mathrm{C}$ for different values of the external field $\mathrm{H}$ applied
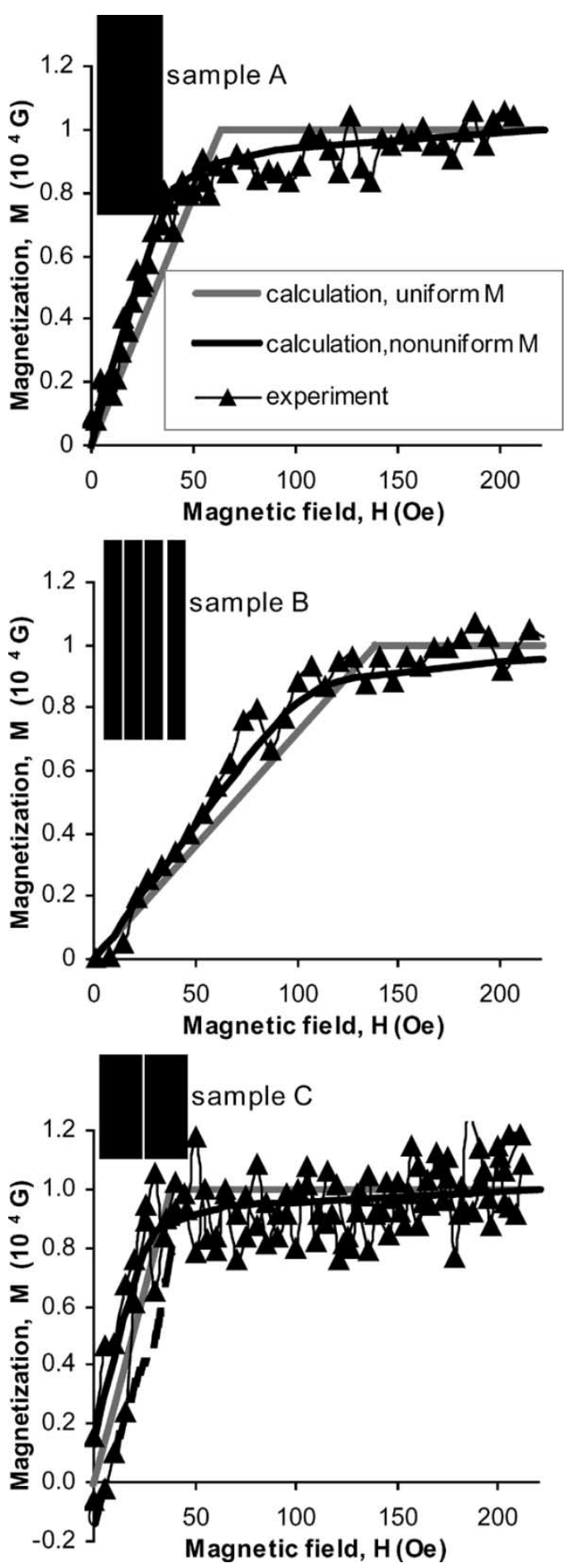

Fig. 1. Hard-axis B-H loop measurement data (markers) versus the self-consistent calculation (solid black lines) and magnetometric approximation (solid gray lines) for three patterned Ni-Fe samples. The dashed black line for sample $\mathrm{C}$ corresponds to the calculated second energy minima.

in the $x$ direction (hard-axis). The regions with (mainly) hard-axis orientation of magnetization are formed near the edges of the sample parallel to the $x$ axis. For segments with a high aspect ratio such as in A and B, the influence of these regions on the overall averaged magnetization is negligible. But for the segments of sample $C$, with a 4:1 aspect ratio, the presence of those regions leads to an observable component 
TABLE I

GeOMETRICAL PARAMETERS OF PATTERNED SAMPLES. EACH SAMPLE WAS Divided Into N Rectangular SEgments, EACH With a LENGTH L

(ALONG THE $z$ AXIS) AND A WIDTH W (ALONG THE $x$ AXIS). THE SEPARATION BETWEEN AdJACENT SAMPLES WAS $3 \mu \mathrm{m}$. THE THICKNESS OF THE FILM WAS $0.5 \mu \mathrm{m}$ IN ALL CASES

\begin{tabular}{c|c|c|c}
\hline Sample & N & L & W \\
\hline A & 1 & $2000 \mu \mathrm{m}$ & $200 \mu \mathrm{m}$ \\
\hline B & 4 & $2000 \mu \mathrm{m}$ & $50 \mu \mathrm{m}$ \\
\hline $\mathrm{C}$ & 2 & $800 \mu \mathrm{m}$ & $200 \mu \mathrm{m}$ \\
\hline
\end{tabular}

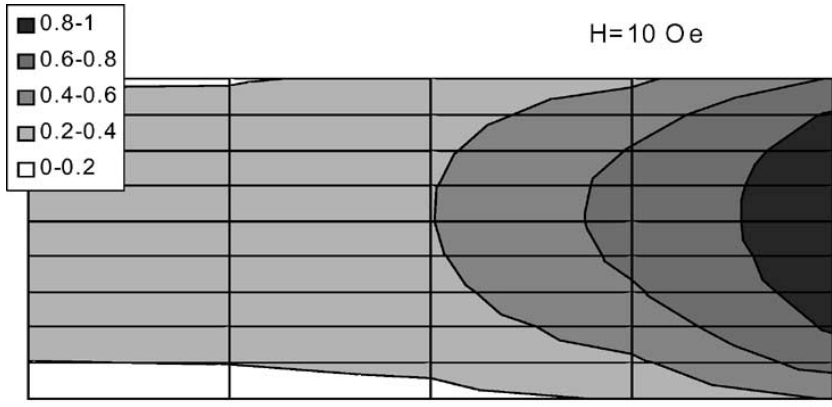

$\mathrm{H}=20 \mathrm{Oe}$

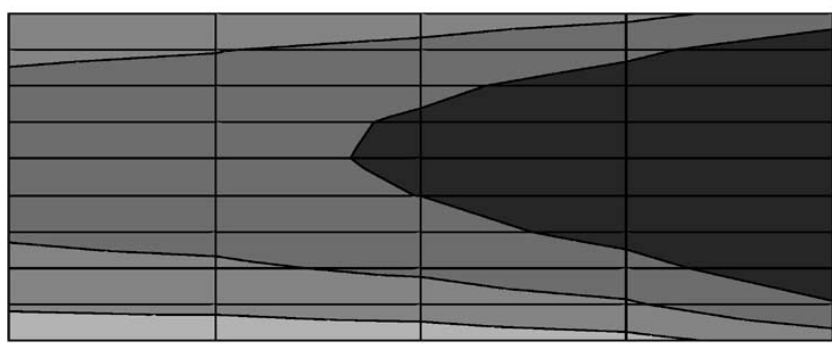

$\mathrm{H}=30 \mathrm{Oe}$

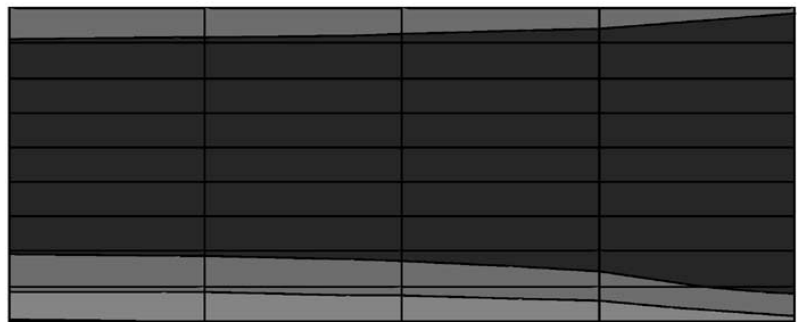

Fig. 2. Distribution of the normalized $x$ component of magnetization $\left(M_{x} / M\right)$ in the sample $\mathrm{C}$ for different values of the external field $H_{\mathrm{DC}}$ applied along the hard axis ( $x$ axis, vertical direction). The sample $\mathrm{C}$ consists of two rectangular segments. Due to symmetry of the solution obtained, here we have only shown the results obtained for half of one of the segment: from the middle to the edge. The results correspond to the upper branch (solid black line) of the magnetization curve (Fig. 1, sample C). of $M$ in the hard direction even in the absence of an external field. It is interesting to note that our self-consistent calculation yields a nonzero magnetization (and two equivalent energy minima) in the absence of the external field for this sample, corresponding to two different orientations of the magnetization near the edges. Whether these states are related to the observed hysteresis in $\mathrm{C}$, or whether they are just numerical artifacts (local instead of global minima of the energy) is still unclear to us. In particular since our model does not take into account domain formation.

\section{CONCLUSION}

The nonuniform distribution of magnetization inside patterned $\mathrm{Ni}-\mathrm{Fe}$ films with rectangular segments has been calculated by minimizing the magnetic energy of the system. The results obtained are in good agreement with experimental data from hard-axis B-H loop measurement. The model can be successfully used for prediction of the FMR frequency and permeability of patterned ferromagnetic cores of on-chip RF devices.

\section{REFERENCES}

[1] M. Yamaguchi, K. Suezawa, M. Baba, K. I. Arai, Y. Shimada, S. Tanabe, and K. Itoh, "Application of bi-directional thin-film micro wire array to RF integrated spiral inductors," IEEE Trans. Magn., vol. 36, pp. 3514-3517, 2000

[2] G. Perrin, J. C. Peuzin, and O. Acher, "Control of the resonance frequency of soft ferromagnetic amorphous thin films by strip patterning," J. Appl. Phys., vol. 8, pp. 5166-5168, 1997.

[3] M. Pardavi-Horvath, J. Yan, and R. Peverley, "Nonuniform internal field in planar ferrite elements," IEEE Trans. Magn., vol. 37, pp. 3881-3884, 2001.

[4] R. J. Joseph and E. Schlomann, "Demagnetizing field in nonellipsoidal bodies," J. Appl. Phys., vol. 36, pp. 1573-1579, 1965.

[5] H. Fukushima, Y. Nakatani, and N. Hayashi, "Volume average demagnetizing tensor of rectangular prisms," IEEE Trans. Magn., vol. 34, pp. 193-198, 1998.

[6] F. C. Stoner and E. P. Wohlfarth, "A mechanism of magnetic hysteresis in heterogeneous alloys," IEEE Trans. Magn., vol. 27, pp. 3475-3518, 1991.

[7] Y. Zhuang, M. Vroubel, B. Rejaei, E. Boellaard, and J. N. Burghartz, "Ferromagnetic RF inductors and transformers for standard CMOS/BiSMOS," in Tech. Dig. IEDM 2002, San Francisco, CA, USA, Dec. 9-11, 2002, pp. 18.06/1-18.06/4.

[8] Y. Zhuang, B. Rejaei, E. Boellaard, M. Vroubel, and J. N. Burghartz, "GHz band-stop microstrip filter using patterned $\mathrm{NiFe}$ ferromagnetic film," IEEE Microwave Wireless Comp. Lett., vol. 12, pp. 473-475, 2002. 